\title{
MicroRNA-101 sensitizes hepatocellular carcinoma cells to doxorubicin-induced apoptosis via targeting Mcl-1
}

\author{
HAIFEI HE ${ }^{1}$, WEI TIAN ${ }^{1,2}$, HAILONG CHEN ${ }^{1,2}$ and YONGCHUAN DENG ${ }^{1,2}$ \\ ${ }^{1}$ Department of Surgical Oncology, The Second Affiliated Hospital; \\ ${ }^{2}$ Cancer Institute (The Key Laboratory of Cancer Prevention and Intervention, China National Ministry of Education), \\ The Second Affiliated Hospital, School of Medicine, Zhejiang University, Hangzhou, Zhejiang 310009, P.R. China
}

Received February 13, 2015; Accepted December 8, 2015

DOI: $10.3892 / \mathrm{mmr} .2015 .4727$

\begin{abstract}
MicroRNAs (miRNAs/miRs) are important regulators of multiple cellular processes, and their dysregulation is a common event in tumorigenesis, including the development of hepatocellular carcinoma (HCC). Studies have shown that certain miRNAs are associated with resistance to chemotherapy or drug sensitization; however, the underlying mechanisms have largely remained elusive. Multiple drug resistance is a major barrier for the treatment of advanced HCC. In the present study, miR-101 was observed to be downregulated in a panel of HCC cell lines, suggesting that it has a tumor suppressor role. Furthermore, transfection of miR-101 significantly enhanced the cytotoxicity of doxorubicin to HepG2 cells. While overexpression of miR-101 did not influence the accumulation of doxorubicin, it promoted the apoptosis-inducing effect of doxorubicin in HepG2 cells. A bioinformatics analysis predicted that miR-101 directly targeted the 3'-untranslated region of myeloid cell leukemia 1 (Mcl-1), which was verified by a luciferase reporter assay. Finally, transfection of HepG2 cells with Mcl-1 expression plasmid inhibited apoptosis caused by doxorubicin plus miR-101 expression. In conclusion, the present study showed that miR-101 is a negative regulator of Mcl-1 in HCC, and the combination of miR-101 expression with doxorubicin may represent a novel approach for the treatment of HCC.
\end{abstract}

\section{Introduction}

Hepatocellular carcinoma (HCC) represents the second most common cause of cancer-associated mortality worldwide due to late diagnosis and poor treatment options (1). Studies have suggested that aberrant regulation of cell death

Correspondence to: Dr Yongchuan Deng, Department of Surgical Oncology, The Second Affiliated Hospital, School of Medicine, Zhejiang University, 88 Jiefang Road, Shangcheng, Hangzhou, Zhejiang 310009, P.R. China

E-mail: zjyongchuandeng@163.com

Key words: microRNA-101, hepatocellular carcinoma, myeloid cell leukemia-1, HepG2, apoptosis, doxorubicin signaling pathways and loss of several tumor suppressors, including apoptotic pathways and tumor suppressor miRNAs (miRNAs/miRs), are involved in the genesis of HCC. However, the molecular pathogenesis of HCC has largely remained elusive (2,3). Although surgery and liver transplantation are able to cure patients with early-stage HCC, chemotherapy is the main treatment option for patients with advanced or unresectable liver cancers (4).

Doxorubcin (DOX), also known as adriamycin, is widely used as an anti-tumor drug. Its mechanisms of action include the induction of apoptosis of tumor cells by intercalating into their DNA to inhibit its transcription and initiate a DNA damage response (5). DOX-based chemotherapy is used against a wide range of cancer types, including ovarian (6), breast (7) and bladder cancer (8) as well as HCC (9). However, the clinical application of DOX is limited due to cardiac toxicity and drug resistance (10). Therefore, it is urgently required to develop novel and efficient approaches to improve the curative effects of DOX for HCC treatment.

The relevance of miRNAs in tumorigenesis and drug resistance of cancer types, including $\mathrm{HCC}$, has become increasingly known $(11,12)$. miRNAs are a class of small, endogenous, non-coding, single-stranded RNAs of 19-25 nucleotides, which either induce mRNA degradation or inhibit mRNA translation via binding with imperfect complementary sequences within the 3'-untranslated region (3'-UTR) of the target mRNA (13). Aberrant expression of miRNAs is associated with tumor development through regulation of their respective target genes. For example, miR-218 was reported to suppress the growth of esophageal squamous cell carcinoma by regulating the PI3K/AKT/mTOR pathway (14). miR-17 and miR-106b are frequently downregulated in the serum of gastric cancer patients, which may therefore be utilized as tumor markers for the early diagnosis of gastric cancer (15). Although miR-101 has been demonstrated to suppress the growth of multiple cancer types, including HCC (16-18), its utilization as a target in chemotherapy has remained to be validated. The present study aimed to assess the role of miR-101 in HCC as well its ability to enhance the chemotherapeutic efficacy of DOX. Furthermore, the underlying mechanism was elucidated by identifying a direct target of miR-101 using bioinformatics analysis and a luciferase reporter assay. The present study indicated that the combination of forced overexpression of 
miR-101 enhanced the apoptosis-inducing effects of doxorubicin via myeloid cell leukemia 1 (Mcl-1), which may represent a novel approach for the treatment of HCC.

\section{Materials and methods}

Cell culture. The HepG2, Hep3B, Huh7 and PLC human HCC cell lines and the $\mathrm{L}-\mathrm{O} 2$ normal liver cell line were obtained from the Institute of Biochemistry and Cell Biology, Chinese Academy of Sciences (Shanghai, China) and cultured in Dulbecco's modified Eagle's medium (DMEM) (Gibco; Thermo Fisher Scientific, Waltham, MA, USA) with $10 \%$ fetal bovine serum (FBS; Gibco) at $37^{\circ} \mathrm{C}$ in a humidified atmosphere containing $5 \% \mathrm{CO}_{2}$.

Reverse-transcription quantitative polymerase chain reaction (RT-qPCR) analysis of miR-101 and Mcl-1 mRNA expression. Total RNA was isolated from cultured cells using TRIzol reagent (Invitrogen; Thermo Fisher Scientific) following the manufacturer's instructions. For analysis of mature miR-101 expression, stem-loop RT-qPCR (19) was employed using Moloney murine leukemia virus Reverse Transcriptase (Invitrogen) following the manufacturer's instructions. The miR-101 RT primer (Ribobio, Guangzhou, China) had the following sequence: 5'-CTCAAC TGGTGTCGTGGAGTCGGCAATTCAGTTGAGATG

TCATG-3'. Relative expression was calculated using the $2^{-\Delta \Delta C q}$ method (20) and normalized to the expression of U6 small RNA. Mcl-1 mRNA was also quantified by RT-qPCR using the PrimeScript RT reagent kit (Takara Bio Inc., Otsu, Japan) for reverse transcription and SYBR ${ }^{\circledR}$ Premix Ex Taq II (Takara Bio, Inc.) for PCR amplification on an Applied Biosystems 7900HT thermocycler (Thermo Fisher Scientific). The PCR reaction mix consisted of $12.5 \mu \mathrm{l} 2 \mathrm{X} \mathrm{SYBR}^{\circledR}$ Premix Ex Taq II, $1 \mu \mathrm{l}$ forward primer $(10 \mu \mathrm{M}), 1 \mu 1$ reverse primer $(10 \mu \mathrm{M}), 2 \mu \mathrm{l} \mathrm{cDNA}$ and $8.5 \mu \mathrm{l}$ $\mathrm{H}_{2} \mathrm{O}$. PCR was performed under the following thermal cycling conditions: $95^{\circ} \mathrm{C}$ for $30 \mathrm{sec}$, followed by 40 cycles of $95^{\circ} \mathrm{C}$ for $5 \mathrm{sec}$ and $60^{\circ} \mathrm{C}$ for $30 \mathrm{sec}$, and one cycle of $95^{\circ} \mathrm{C}$ for $15 \mathrm{sec}, 60^{\circ} \mathrm{C}$ for $60 \mathrm{sec}$ and $95^{\circ} \mathrm{C}$ for $15 \mathrm{sec}$ for dissociation. PCR primers were obtained from Ribobio and had the following sequences: Mcl-1 forward, 5'-TGGCTAAACACTTGAAGACC-3' and reverse, 5'-GGAAGAACTCCACAAACCC-3'; $\beta$-actin forward, 5'-CAGAGCCTCGCCTTTGCC-3' and reverse, 5'-GTCGCC CACATAGGAATC-3'. After the products were purified using the MiniBEST DNA Fragment Purification kit (cat. no. 9761; Takara Bio, Inc.) according to the manufacturer's instructions, the PCR products were quantified by reading the absorbance at $260 \mathrm{~nm}$ PCR products. The expression levels were normalized to $\beta$-actin.

Plasmid construction. Bioinformatics analysis using TargetScan (http://www.targetscan.org/) indicated that Mcl-1 represents a target gene of miR-101. To verify this, luciferase reporter vectors containing a wild-type (W) or mutated (M) fragment of the 3'-UTR of Mcl-1 were constructed. The 3'-UTR of Mcl-1 was amplified by PCR using cDNA from HepG2 cells as a template. The Mcl-1 3'UTR and open reading frame fragment were amplified by PCR. The following primers were utilized: Mcl-1 3'UTR: Mcl-1-SpeI, 5'-CGACTAGTGCAACAAACA AACTTTGTTTG-3' and Mcl-1-HindIII, 5'-CGAAGCTTG CAAAGTTCAAAAGGGTATGA-3'; Mcl-1 open reading frame: Mcl-1-HindIII, 5'-CGAAGCTTATGTTTGGCCTCA
AAAGAAAC-3' and Mcl-1 EcoRI, 5'-CGGAATTCTGTC TTATTAGATATGCCAAACCAG-3'. The reaction conditions were as follows: $95^{\circ} \mathrm{C}$ for $5 \mathrm{~min} ; 35$ cycles of $95^{\circ} \mathrm{C}$ for $45 \mathrm{sec}$, $58^{\circ} \mathrm{C}$ for $45 \mathrm{sec}$ and $72^{\circ} \mathrm{C}$ for $60 \mathrm{sec}$; and $72^{\circ} \mathrm{C}$ for $5 \mathrm{~min}$. PCR products and the vector were then digested with SpeI/HindIII or HindIII/EcoRI (Takara Bio, Inc.). To ligate the Mcl-1 fragment into the vector, the digestion products were incubated with T4 DNA Ligase (Takara Bio, Inc.). The resulting recombinant plasmid was transformed into E. coli (DH5 strain; Tiangen Co., Ltd., Beijing, China) for replication and then extracted from E. coli using an EndoFree Plasmid Maxi kit (Qiagen, Hilden, Germany). Total RNA was isolated from cultured cells using TRIzol reagent, and the cDNA library was synthesized by using the PrimeScript RT reagent kit (Takara Bio, Inc.) according to the manufacturer's instructions. The obtained nucleotides were cloned into the SpeI/HindIII site of the pMIR vector (pMIR-REPORT ${ }^{\mathrm{TM}}$ miRNA Expression Reporter Vector System; Ambion; Thermo Fisher Scientific). For cloning, $1 \mu 1$ restriction enzymes (Takara Bio, Inc.), $2 \mu \mathrm{l}$ 10X buffer (Takara Bio, Inc.) and $1 \mu \mathrm{g}$ DNA were used with $\mathrm{H}_{2} \mathrm{O}$ added for a total volume of $20 \mu \mathrm{l}$. The recombinant plasmid was named pMIR-Mcl-1-W. The mutant plasmid, pMIR-Mcl-1-M, was created by mutating the seed regions of the miR-101 binding sites (CAGUACUGUA) by using a site-directed mutagenesis kit (Takara Bio, Inc.).

To construct an Mcl-1 overexpression vector, the Mcl-1 open reading frame without the 3'-UTR was amplified by PCR with HepG2 cDNA as a template and cloned into the HindIII/EcoRI site of the pcDNA3.1 vector (Invitrogen) with the respective restriction enzymes (Takara Bio, Inc.) according to the abovementioned procedure; the resulting recombinant plasmid was named pcDNA-Mcl-1.

Transient transfection. HepG2 cells were seeded into 12-well plates and transfected at $\sim 80 \%$ confluence. The Firefly luciferase reporters (pMIR-Mcl-1-W or pMIR-Mcl-1-M; $2 \mu \mathrm{g} / \mathrm{ml}$ ), Renilla luciferase pRL-TK vector (100 ng/ml; Promega Corp., Madison, WI, USA), eukaryotic expression vector (pcDNA3.1 or pcDNA-Mcl-1; $2 \mu \mathrm{g} / \mathrm{ml}$ ), RNA oligonucleotides [negative control oligonucleotide (NCO; 5'-GUACUAACCUAU GUGAAAUAG-3'; Genepharma Co., Shanghai, China), miR-101 mimics (5'-UACAGUACUGUGAUAACUGAA-3'; Genepharma Co.) or multidrug restistance 1 (MDR1) small interfering (si)RNA (5'-GGAAAAGAAACCAACUGU CUU-3'; $50 \mathrm{nM}$; Ribobio)], were transiently transfected into the HepG2 cells with Lipofectamine 2000 reagent (Invitrogen) following the manufacturer's instructions.

Luciferase assay. HepG2 cells were seeded into 48-well plates at $80 \%$ cell confluence, followed by transfection with the Firefly luciferase reporters pMIR plus the Renilla luciferase pRL-TK vector in combination with miR-101-mimics and NCO using Lipofectamine 2000. Luciferase activity was measured $48 \mathrm{~h}$ after transfection by using the Dual-Luciferase Reporter assay system (Promega Corp.) according to the manufacturer's instructions. Firefly luciferase activity was normalized to Renilla luciferase activity for each transfected well.

Measurement of cell viability by 3-(4,5-dimethylthiazol-2-yl)-2,5-diphenyltetrazolium bromide (MTT). The effect 
A

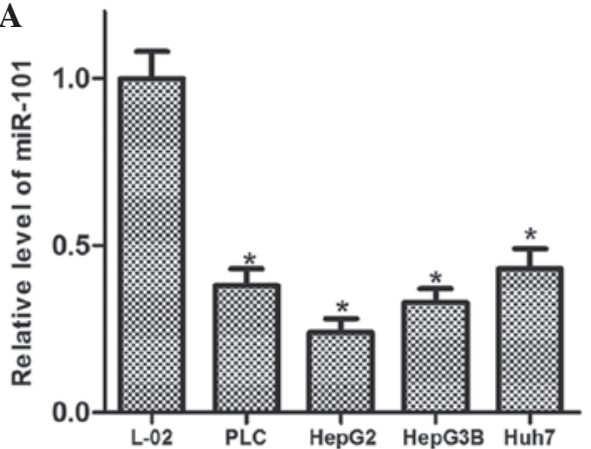

B

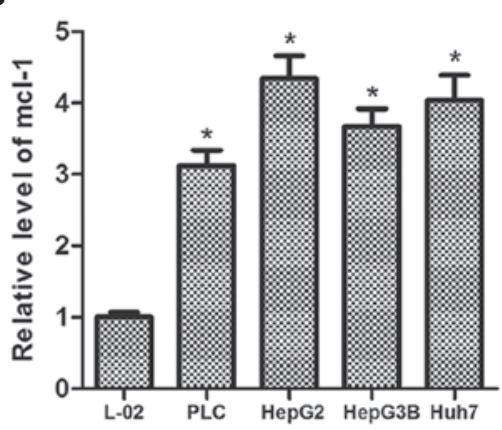

C

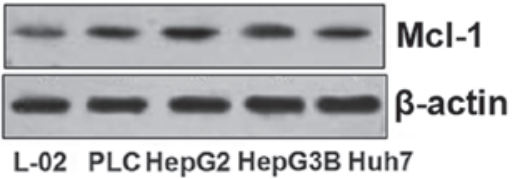

Figure 1. Inverse correlation of miR-101 and Mcl-1 in HCC cell lines. (A) Expression of miR-101 was down-regulated in HCC cell lines according to RT-qPCR analysis. (B) Mcl-1 expression was significantly upregulated in HCC cell lines compared with levels in the normal liver cell line L-O2 according to RT-qPCR analysis. Values are expressed as the mean \pm standard deviation. ${ }^{*} \mathrm{P}<0.05$ vs. L-O2. (C) Western blot representative of three experiments showing that Mcl-1 was obviously upregulated in HCC cell lines compared with levels in L-O2 cells. miR, microRNA; HCC, hepatocellular cancer; Mcl-1, myeloid cell leukemia 1; RT-qPCR, reverse-transcription quantitative polymerase chain reaction.

of miR-101, DOX (Sigma-Aldrich, St. Louis, MO, USA) and their combination on the viability of HepG2 cells was measured using an MTT assay. HepG2 cells were seeded in triplicate in a 96 -well plate at a density of $5 \times 10^{3}$ per well. Following culture for $12 \mathrm{~h}$, cells were transfected with miR-101 or NCO. Following incubation for $24 \mathrm{~h}$, cells were treated with various concentrations of DOX $(0.1,0.25,0.5,1,1.5$ and $2 \mu \mathrm{g} / \mathrm{ml})$ for $48 \mathrm{~h}$. The inhibition of cell viability was detected using the MTT assay as described previously using MTT and dimethyl sulfoxide (Sigma-Aldrich) (21). The absorbance values of wells from the various treatment groups at $570 \mathrm{~nm}$ were determined using a SpectraMax M5 (Molecular Devices, Sunnyvale, CA, USA) and compared with the absorbance of the control cells to calculate the relative cell viability.

Doxorubicin accumulation. HepG2 cells were transfected with miR-101 mimics, NCO or MDR1 siRNA for $24 \mathrm{~h}$, and the cells were then treated with $0.25 \mu \mathrm{g} / \mathrm{ml}$ doxorubicin. After $2 \mathrm{~h}$ of incubation, the cells were washed three times with phosphate-buffered saline and the mean fluorescence intensity of intracellular DOX was determined using flow cytometry (FACSCalibur; BD Biosciences, Franklin Lakes, NJ, USA) at an excitation wavelength of $488 \mathrm{~nm}$ and an emission wavelength of $575 \mathrm{~nm}$.

Western blot analysis. Total cellular extracts were prepared with radioimmunoprecipitation assay lysis buffer (Cell Signaling Technology, Inc., Danvers, MA, USA). The protein concentration was determined using the bicinchoninic acid assay (Pierce Biotechnology, Inc., Rockford, IL, USA) and equal amounts of protein $(50 \mu \mathrm{g})$ were separated by $12.5 \%$ sodium dodecyl sulfate polyacrylamide gel electrophoresis (Sigma-Aldrich). Proteins were then transferred onto polyvinylidene difluoride membranes (Millipore, Billerica, MA, USA) by electroblotting. Non-specific binding was blocked using 5\% (w/v) skimmed milk in Tris-buffered saline with 1\% Tween-20 (TBST) for $2 \mathrm{~h}$ at room temperature. The membranes were then incubated with the primary antibodies rabbit anti- $\beta$-actin monoclonal antibody (mAb) (cat. no. 4970; 1:1,000 dilution; Cell Signaling Technology, Inc.) and Mcl-1 rabbit mAb (cat. no. 5453; 1:1,000 dilution; Cell Signaling Technology, Inc.) overnight at $4^{\circ} \mathrm{C}$. Following several washes with TBST, the membranes were incubated in horseradish peroxidase-conjugated goat anti-rabbit immunoglobulin G (cat no. 7074; 1:2,000 dilution; Cell Signaling Technology, Inc.) for $2 \mathrm{~h}$ at room temperature and were then washed five times with TBST for $10 \mathrm{~min}$ each. Blots were visualized using an enhanced chemiluminescence detection kit (cat. no. 32106; Pierce Biotechnology, Inc.) and images were captured using Gel Doc ${ }^{\mathrm{TM}}$ EZ (Bio-Rad Laboratories, Inc., Hercules, CA, USA).

Measurement of apoptosis. DOX was added to the cell medium at a final concentration of $0.25 \mu \mathrm{g} / \mathrm{ml}$ at $24 \mathrm{~h}$ after transfection with miR-101 mimics. After $24 \mathrm{~h}$ of incubation, cells were collected, stained with Annexin V/propidium iodide (PI) (Sigma-Aldrich, St. Louis, MO, USA) for $15 \mathrm{~min}$ at room temperature according to the manufacturer's instructions and analyzed using flow cytometry (FACSCalibur).

Statistical analysis. Statistical analysis was performed using SPSS 15.0 software (SPSS, Inc., Chicago, IL, USA). Student's t-test and analysis of variance were used to compare mean values. Values are expressed as the mean \pm standard deviation and derived from at least three independent experiments. $\mathrm{P}<0.05$ was considered to indicate a statistically significant difference between values.

\section{Results}

miR-101 is downregulated and Mcl-1 is upregulated in HCC.To evaluate the role of miR-101 in HCC cells as well as any mechanistic association with Mcl-1, the expression levels of miR-101 and Mcl-1 were determined in the L-O2 normal liver cell line as well as in four HCC cell lines (PLC, HepG2, Hep3B and Huh7). The results showed that miR-101 expression was reduced in the human HCC cell lines compared with that in L-O2 cells (Fig. 1A). Conversely, Mcl-1 was significantly upregulated in all four liver cancer cell lines at the mRNA as well as at the protein level (Fig. 1B and C). These results suggested that miR-101 exerts a tumor suppressor role in $\mathrm{HCC}$, and that the expression of Mcl-1 is involved in liver carcinogenesis.

miR-101 sensitizes HCC cells to DOX. The viability of HepG2 cells treated with various concentrations of DOX for $48 \mathrm{~h}$ was 
A

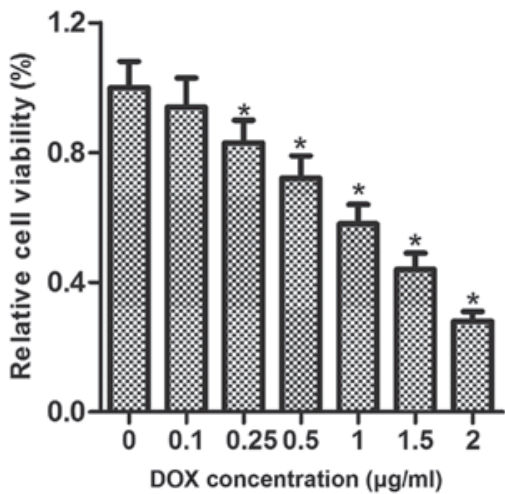

B

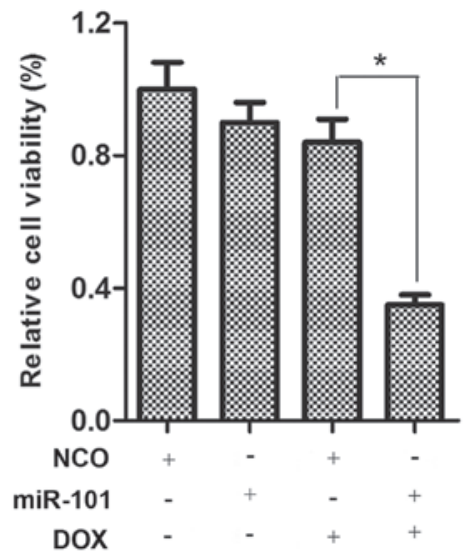

Figure 2. miR-101 increases the cytotoxic effects of DOX in HepG2 cells. (A) Cell viability was measured using an MTT assay after 48 h of DOX treatment. ${ }^{*} \mathrm{P}<0.05$ vs. control group. (B) Enforced vector-mediated expression of miR-101 enhanced the cytotoxicity of DOX in HepG2 cells compared with that in cells treated with DOX or transfected with miR-101 only. ${ }^{*} \mathrm{P}<0.05$. Values are expressed as the mean \pm standard deviation. DOX, doxorubicin; miR, microRNA; NCO, negative control oligonucleotide.
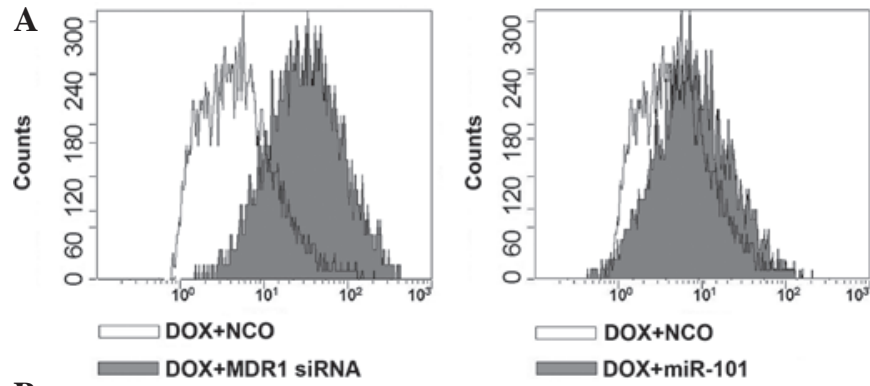

B

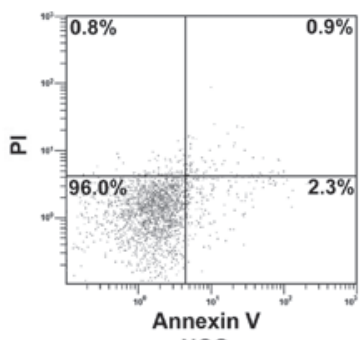

NCO
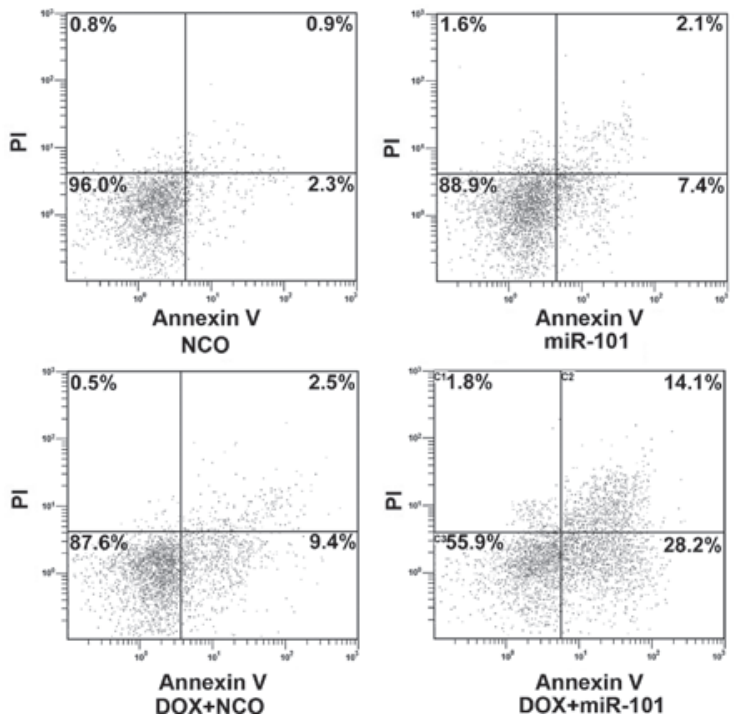

miR-101

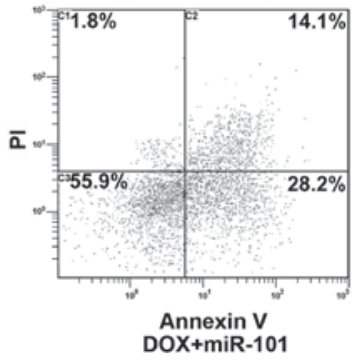

Figure 3. miR-101 enhances the apoptotic effects of DOX without influencing DOX accumulation. (A) HepG2 cells were transfected with NCO, miR-125b or MDR1 siRNA for $24 \mathrm{~h}$, followed by incubation with $0.25 \mu \mathrm{g} / \mathrm{ml}$ DOX for another $2 \mathrm{~h}$. The accumulation of DOX was evaluated by flow cytometric analysis. (B) miR-101 or NCO was transfected into HepG2 cells for $24 \mathrm{~h}$, followed by incubation with/without $0.25 \mu \mathrm{g} / \mathrm{ml} \mathrm{DOX}$ for another $24 \mathrm{~h}$. The apoptotic rate was evaluated by flow cytometric analysis. Dot plots representative of three independent experiments are shown. siRNA, small interfering RNA; miR, microRNA; DOX, doxorubicin; NCO, negative control oligonucleotide; PI, propidium iodide.

determined using an MTT assay, demonstrating that DOX inhibited the cell viability in a dose-dependent manner (Fig. 2A). To examine the effects of miR-101 on the viability of HCC cells,
HepG2 cells were transfected with miR-101 mimics for $24 \mathrm{~h}$ and treated with DOX at a moderate concentration $(0.25 \mu \mathrm{g} / \mathrm{ml})$ for $48 \mathrm{~h}$. As shown in Fig. 2B, the viability of HepG2 cells treated with DOX plus miR-101 was considerably lower compared to that of cells treated with DOX or miR-101 mimics alone.

miR-101 does not affect the accumulation of DOX in HCC cells. To determine the underlying mechanisms of miR-101-mediated sensitization of HCC cells to DOX, the present study assessed the effects of miR-101 mimics on DOX accumulation in HepG2. Cells transfected with MDR1 (encoding P-glycoprotein) siRNA to increase the accumulation of DOX (22) were used as the positive control. As expected, transfection of MDR1 siRNA significantly increased the uptake of DOX in HepG2 cells (Fig. 3A). However, the accumulation of DOX in HepG2 cells was not influenced by transfection with miR-101 mimics, suggesting that a mechanism other than the enhancement of drug accumulation is responsible for the synergism of miR-101 and DOX.

miR-101 enhances apoptosis induction by DOX. To further investigate the underlying mechanisms of miR-101-mediated sensitization of HCC cells to DOX, the apoptosis-inducing effects of miR-101, DOX and their combination were assessed by Annexin V/PI staining and flow cytometric analysis. As shown in Fig. 3B, a marked increase of HepG2-cell apoptosis was observed upon combined treatment with DOX and miR-101 compared with that in the single treatment groups. These results suggested that the overexpression of miR-101 sensitizes HCC cells to DOX through enhancing the apoptotic rate.

miR-101 negatively regulates $\mathrm{Mcl}-1$ expression in $\mathrm{HCC}$ cells. To explore the regulatory mechanisms of miR-101, the public miRNA database TargetScan was used for prediction of its target genes, which suggested Mcl-1 as a possible target. To determine the regulation of Mcl-1 expression by miR-101, miR-101 mimics were transfected into HepG2 cells and the effects on Mcl-1 expression were assessed by RT-qPCR and western blot analysis. As shown in Fig. 4A, transfection with miR-101 mimics significantly enhanced the 
A

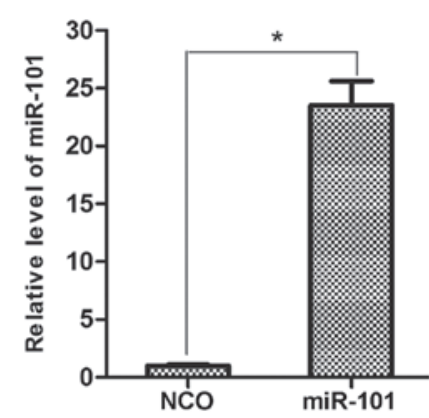

B

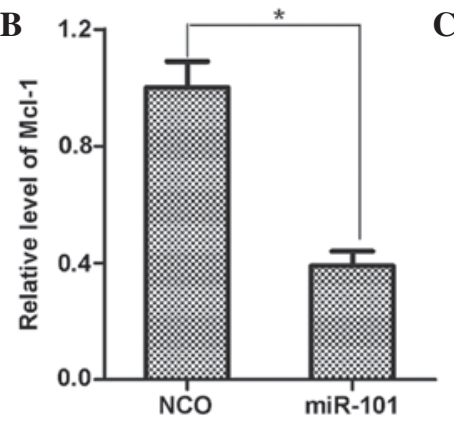

C

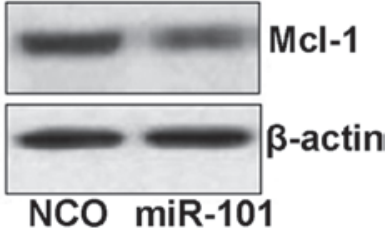

Figure 4. Inverse correlation between Mcl-1 and miR-101 in HepG2 cells. (A) HepG2 cells were transfected with miR-101 or NCO for 6 h and the miR-101 levels were then detected by RT-qPCR analysis. (B) Expression of Mcl-1 in HepG2 was examined by RT-qPCR after transfection with miR-101 mimics or NCO. Values are expressed as the mean \pm standard deviation. "P<0.05. (C) Western blot analysis of Mcl-1 protein levels in HepG2 cells treated as above. A blot representative of three experiments is shown. miR, microRNA; Mcl-1, myeloid cell leukemia 1; RT-qPCR, reverse-transcription quantitative polymerase chain reaction; $\mathrm{NCO}$, negative control oligonucleotide.

A

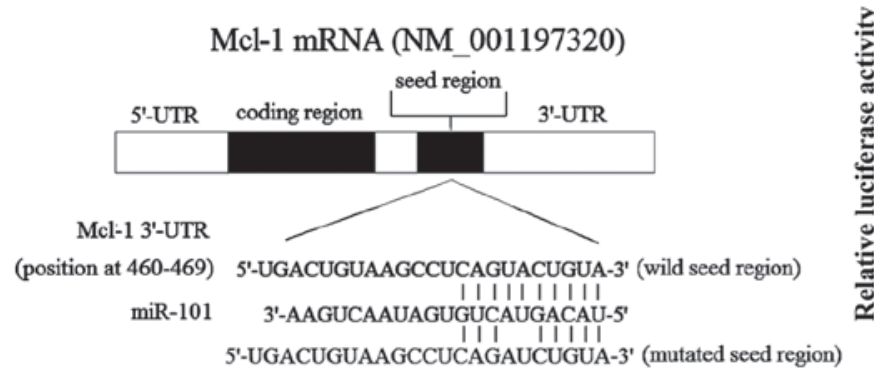

B

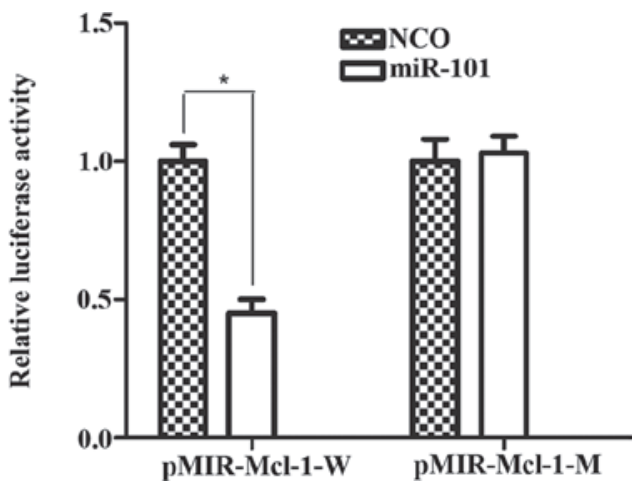

Figure 5. Mcl-1 is ta direct target of miR-101. (A) TargetScan analysis predicted that Mcl-1 was a potential target of miR-101. (B) A dual luciferase assay was performed in HepG2 cells co-transfected with RNA mimics as well as pRL-TK and pMIR plasmids containing the wild-type or mutant 3'-UTR of the Mcl-1 gene. The activity of Firefly luciferase expressed by pMIR of each sample was normalized to the Renilla luciferase activity. Values are expressed as the mean \pm standard deviation. *P<0.05. miR, microRNA; Mcl-1, myeloid cell leukemia 1; NCO, negative control oligonucleotide; UTR, untranslated region; W, wild-type; M, mutated.
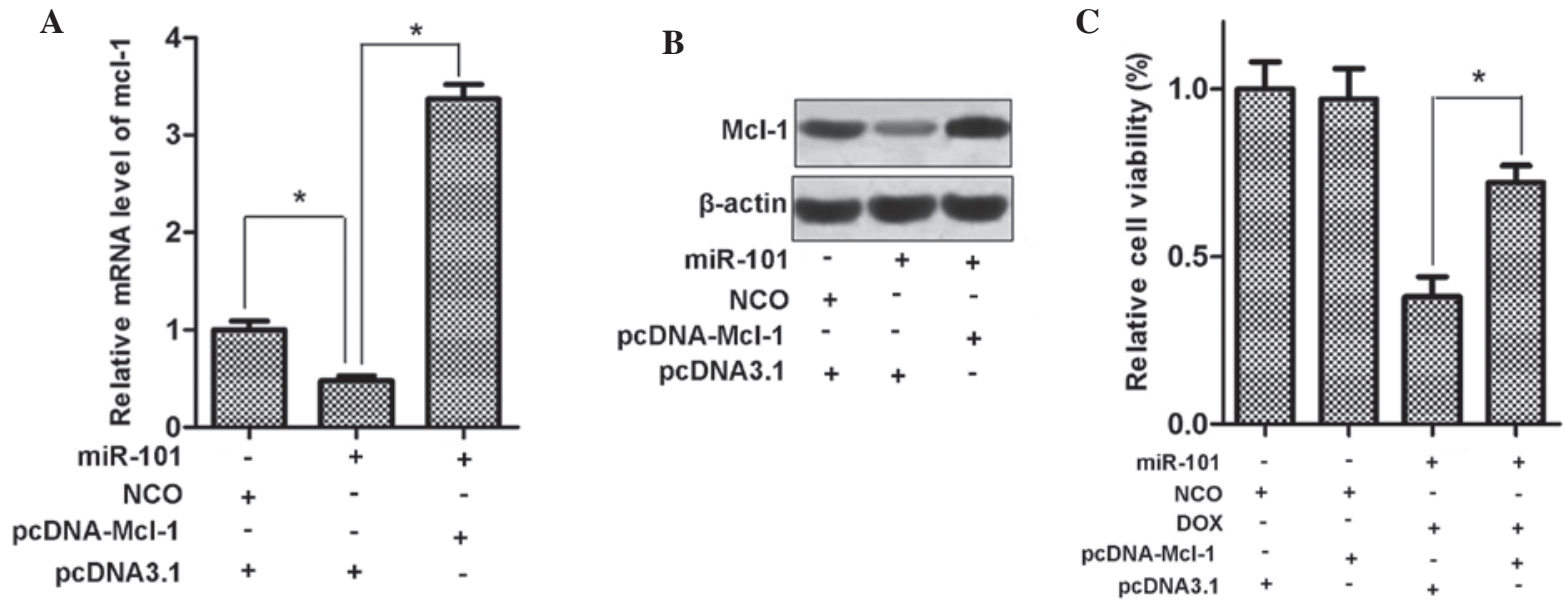

Figure 6. Mcl-1 is involved in miR-101-enhanced cytotoxicity of doxorubicin. (A) HepG2 cells were co-transfected with miR-101 and pcDNA-Mcl-1 for 24 h, and then the mRNA expression of Mcl-1 was detected by reverse-transcription quantitative polymerase chain reaction analysis. (B) Protein levels of Mcl-1 in HepG2 cells treated as described above were assessed by western blot analysis. A blot representative of three experiments is shown. (C) HepG2 cells were co-transfected with pcDNA-Mcl-1 and miR-101 for $24 \mathrm{~h}$, followed by incubation with $0.25 \mu \mathrm{g} / \mathrm{ml}$ DOX for another $24 \mathrm{~h}$. An MTT assay was performed to evaluate the cell viability. Values are expressed as the mean \pm standard deviation. ${ }^{*} \mathrm{P}<0.05$. miR, microRNA; Mcl-1, myeloid cell leukemia 1; NCO, negative control oligonucleotide; DOX, doxorubicin.

levels of miR-101 in HepG2 cells compared to those in the NCO-transfected group. Furthermore, consistent with the bioinformatics prediction, ectopically expressed miR-101 decreased the expression of Mcl-1 at the mRNA as well as at the protein level (Fig. 4B and C). This result suggested that Mcl-1 expression is regulated by miR-101 in HCC cells. 
Mcl-1 is a direct target of miR-101. As illustrated in Fig. 5A, Mcl-1 contains a putative miR-101 target site in its 3'-UTR (5'-CAGUACUGUA-3'; nucleotides 460-469 of Mcl-1 mRNA 3 '-UTR) and is therefore a potential target of miR-101. To confirm whether miR-101 directly regulates Mcl-1 expression, the target sequence of the Mcl-1 3'-UTR or a mutant sequence was cloned into the pMIR luciferase reporter vector, which was then respectively transfected into HepG2 cells in combination with miR-101 mimics. The results showed that co-transfection with miR-101 significantly suppressed the luciferase activity of the reporter plasmid containing the wild-type 3'-UTR of Mcl-1 compared with transfection with NCO, while the luciferase activity of the reporter plasmid carrying the mutant 3'-UTR remained unaffected by the miR-101 mimics (Fig. 5B). All these results confirmed that Mcl-1 is a direct target of miR-101 in HepG2 cells.

Mcl-1 mediates the sensitization of HCC cells to DOX by $m i R-101$. Since miR-101 sensitized HepG2 cells to DOX and Mcl-1 is a direct target of miR-101, the present study examined whether eukaryotic expression vector-mediated upregulation of Mcl-1 reverses the cytotoxic effects of DOX plus miR-101 in HepG2 cells. First, HepG2 cells were transfected with pcDNA3.1 carrying with the open reading frames of the Mcl-1 gene, and Mcl-1 expression was evaluated at the mRNA and protein level. As shown in Fig. 6A and B, transfection with pcDNA-Mcl-1 significantly increased the expression of Mcl-1 in HepG2 cells, even in the presence of miR-101 mimics. Furthermore, as expected, exogenous expression of Mcl-1 significantly decreased the cytotoxicity of DOX combined with miR-101 in HepG2 cells (Fig. 6C). These results supported the notion that Mcl-1 is involved in the sensitization of HCC cells to doxorubicin by miR-101.

\section{Discussion}

It has been demonstrated that miRNAs are involved in carcinogenesis through regulating the expression of a large variety of genes, including oncogenes and tumor suppressor genes (23). It has been reported that dysregulation of miR-101 is associated with tumorigenesis, development and invasion, which is essential in multiple cancer types, including bladder cancer (24) and human hepatocellular carcinoma $(25,26)$. Furthermore, it has bee reported that overexpression of miR-101 induced apoptosis in gastric cancer cells (27). The present study demonstrated that the expression of miR-101 was reduced in four human HCC cell lines compared with that in a normal liver cell line, illustrating that miR-101 acts as a tumor suppressor in liver cancer. By contrast, Mcl-1 was significantly upregulated in all four liver cancer cell lines. These results inferred that miR-101 and Mcl-1 are inversely correlated in HCC.

Mcl-1 is a key anti-apoptotic protein of the B-cell lymphoma 2 family, which controls apoptosis (28). In addition, Mcl-1 has been implicated in resistance to chemotherapy in multiple cancer types. Sun et al (29) demonstrated that siRNA-mediated inhibition of Mcl-1 effectively increased the sensitivity of human colon cancer cells to ABT-737-induced apoptosis, while apoptosis was significantly attenuated by enforced expression of Mcl-1. As high expression of Mcl-1 contributes to the ability of cancer cells to survive and evade apoptosis induced by chemotherapeutic drugs, high levels of Mcl-1 protein have been correlated with poor prognosis of various cancer types $(30,31)$.

The present study found that miR-101 significantly increased the cytotoxic effects of DOX in HepG2 cells. Flow-cytometric analysis revealed that the accumulation of DOX in HepG2 cells was not influenced by miR-101, suggesting that other pathways are responsible for the observed synergism of miR-101 and DOX. It was then demonstrated that miR-101 enhanced the anti-cancer effects of DOX by increasing its ability to induce apoptosis in HepG2 cells. Bioinformatics analysis revealed that Mcl-1 was a putative target of miR-101. Since downregulation of Mcl-1 is a mechanism of apoptosis induction (32), the present study determined the effects of miR-101 mimics on Mcl-1 in HepG2 cells, revealing that upregulation of miR-101 expression significantly reduced Mcl-1 expression. Furthermore, a luciferase reporter assay proved that miR-101 directly targeted the Mcl-1 3'-UTR. Of note, enforced expression of Mcl-1 by transfection of pcDNA-Mcl-1 markedly reduced the effects of miR-101 on the sensitivity of HCC cells to DOX, suggesting that miR-101 enhances the sensitivity of HCC cells to DOX via Mcl-1. It is therefore concluded that miR-101 is involved in DOX resistance of HCC through regulating Mcl-1 expression.

In conclusion, the results of the present study demonstrated that miR-101 was downregulated in HCC cell lines, while its overexpression enhanced the sensitivity of HepG2 cells to the chemotherapeutic agent DOX by facilitating apoptosis. Of note, Mcl-1 was confirmed as a functional target of miR-101 in HCC, demonstrating that miR-101 may enhance the sensitivity of cancer cells by downregulating Mcl-1 expression. These results suggested the potential application of miR-101 overexpression as an adjuvant in DOX-based therapy.

\section{Acknowledgements}

The present study was supported by the Key Laboratory of Cancer Prevention and Intervention, China National Ministry of Education.

\section{References}

1. El-Serag Hb: Hepatocellular carcinoma. N Engl J. Med 365: 1118-1127, 2011.

2. Lyra-González I, Flores-Fong LE, González-García I, Medina-Preciado D and Armendáriz-Borunda J: MicroRNAs dysregulation in hepatocellular carcinoma: Insights in genomic medicine. World J Hepatol 7: 1530-1540, 2015.

3. Zhu Z, Zhang X, Wang G and Zheng H: Role of MicroRNAs in hepatocellular carcinoma. Hepat Mon 14: e18672, 2014.

4. Chung V: Systemic therapy for hepatocellular carcinoma and cholangiocarcinoma. Surg Oncol Clin N Am 24: 187-198, 2015.

5. Yang F, Teves SS, Kemp CJ and Henikoff S: Doxorubicin, DNA torsion, and chromatin dynamics. Biochim Biophys Acta 1845: 84-89, 2014

6. Marczak A, Denel-Bobrowska M, Rogalska A, Łukawska M and Oszczapowicz I: Cytotoxicity and induction of apoptosis by formamidinodoxorubicins in comparison to doxorubicin in human ovarian adenocarcinoma cells. Environ Toxicol Pharmacol 39: 369-383, 2015.

7. Xu F, Wang F, Yang T, Sheng Y, Zhong T and Chen Y: Differential drug resistance acquisition to doxorubicin and paclitaxel in breast cancer cells. Cancer Cell Int 14: 538, 2014.

8. Teply BA and Kim JJ: Systemic therapy for bladder cancer-a medical oncologist's perspective. J Solid Tumors 4: 25-35, 2014.

9. Zhao X, Chen Q, Liu W, Li Y, Tang H, Liu X and Yang X: Codelivery of doxorubicin and curcumin with lipid nanoparticles results in improved efficacy of chemotherapy in liver cancer. Int J Nanomedicine 10: 257-270, 2014. 
10. Shuhendler AJ, Prasad P, Zhang RX, Amini MA, Sun M, Liu PP, Bristow RG, Rauth AM and Wu XY: Synergistic nanoparticulate drug combination overcomes multidrug resistance, increases efficacy and reduces cardiotoxicity in a nonimmunocompromised breast tumor model. Mol Pharm 11: 2659-2674, 2014.

11. Ohtsuka M, Ling H, Doki Y, Mori M and Calin GA: MicroRNA processing and human cancer. J Clin Med 4: 1651-1667, 2015.

12. Donzelli S, Mori F, Biagioni F, Bellissimo T, Pulito C, Muti P, Strano $S$ and Blandino G: MicroRNAs: Short non-coding players in cancer chemoresistance. Mol Cell Ther 2: 16, 2014.

13. Winter J, Jung S, Keller S, Gregory RI and Diederichs S: Many roads to maturity: MicroRNA biogenesis pathways and their regulation. Nat Cell Biol 11: 228-234, 2009.

14. Tian H, Hou L, Xiong YM, Huang JX, She YJ, Bi XB and Song XR: MiR-218 suppresses tumor growth and enhances the chemosensitivity of esophageal squamous cell carcinoma to cisplatin. Oncol Rep 33: 981-989, 2015.

15. Zeng Q, Jin C, Chen W, Xia F, Wang Q, Fan F, Du J, Guo Y, Lin C, Yang K, et al: Downregulation of serum miR-17 and miR-106b levels in gastric cancer and benign gastric diseases. Chin J Cancer Res 26: 711-716, 2014.

16. Shen Q, Bae HJ, Eun JW, et al: MiR-101 functions as a tumor suppressor by directly targeting nemo-like kinase in liver cancer. Cancer Lett 344: 204-211, 2014.

17. Lv P, Zhang P, Li X and Chen Y: Micro ribonucleic acid (RNA)-101 inhibits cell proliferation and invasion of lung cancer by regulating cyclooxygenase-2. Thorac Cancer 6: 778-784, 2015

18. Li JT, Jia LT, Liu NN, Zhu XS, Liu QQ, Wang XL, Yu F, Liu YL, Yang AG and Gao CF: MiRNA-101 inhibits breast cancer growth and metastasis by targeting CX chemokine receptor 7 . Oncotarget 6: 30818-30830, 2015.

19. Chen C, Ridzon DA, Broomer AJ, Zhou Z, Lee DH, Nguyen JT, Barbisin M, Xu NL, Mahuvakar VR, Andersen MR, et al: Real-time quantification of microRNAs by stem-loop RT-PCR. Nucleic Acids Res 33: e179, 2005.

20. Livak KJ and Schmittgen TD: Analysis of relative gene expression data using real-time quantitative PCR and the 2(-Delta Delta C(T)) Method. Methods 25: 402-408, 2001.
21. Tong SJ, Liu J, Wang X and Qu LX: MicroRNA-181 promotes prostate cancer cell proliferation by regulating DAX-1 expression. Exp Ther Med 8: 1296-1300, 2014.

22. Misra R, Das M, Sahoo BS and Sahoo SK: Reversal of multidrug resistance in vitro by co-delivery of MDR 1 targeting siRNA and doxorubicin using a novel cationic poly (lactide-co-glycolide) nanoformulation. Int J Pharm 475: 372-384, 2014.

23. Takasaki S: Roles of microRNAs in cancers and development. Methods Mol Biol 1218: 375-413, 2015.

24. Hu Z, Lin Y, Chen H, Mao Y, Wu J, Zhu Y, Xu X, Xu X, Li S, Zheng $X$ and Xie L: MicroRNA-101 suppresses motility of bladder cancer cells by targeting c-Met. Biochem Biophys Res Commun 435: 82-87, 2013.

25. Chiang CW, Huang Y, Leong KW, Chen LC, Chen HC, Chen SJ and Chou CK: PKCalpha mediated induction of miR-101 in human hepatoma HepG2 cells. J Biomed Sci 17: 35, 2010.

26. Zhang Y, Guo X, Xiong L, Kong X, Xu Y, Liu C, Zou L, Li Z, Zhao J and Lin N: MicroRNA-101 suppresses SOX9-dependent tumorigenicity and promotes favorable prognosis of human hepatocellular carcinoma. FEBS Lett 586: 4362-4370, 2012.

27. Wang HJ, Ruan HJ, He XJ, et al: MicroRNA-101 is downregulated in gastric cancer and involved in cell migration and invasion. Eur J Cancer 46: 2295-2303, 2010.

28. Quinn BA, Dash R, Azab B, et al: Targeting Mcl-1 for the therapy of cancer. Expert Opin Investig Drugs 20: 1397-1411, 2011.

29. Sun JG, Xiang J, Zeng XL, Li X, Wu P, Fung KP and Liu FY: Clitocine induces apoptosis and enhances the lethality of ABT-737 in human colon cancer cells by disrupting the interaction of Mcl-1 and Bak. Cancer Lett 355: 253-263, 2014

30. Luo L, Zhang T, Liu H, Lv T, Yuan D, Yao Y, Lv Y and Song Y. MiR-101 and Mcl-1 in nonsmall-cell lung cancer: Expression profile and clinical significance. Med Oncol 29: 1681-1686, 2012.

31. Zhang T, Zhao C, Luo L, Zhao H, Cheng J and Xu F: The expression of Mcl-1 in human cervical cancer and its clinical significance. Med Oncol 29: 1985-1991, 2012.

32. Doi K, Gowda K, Liu Q, Lin JM, Sung SS, Dower C, Claxton D, Loughran TP Jr, Amin S and Wang HG: Pyoluteorin derivatives induce Mcl-1 degradation and apoptosis in hematological cancer cells. Cancer Biol Ther 15: 1688-1699, 2014. 